Check for updates

Cite this: RSC Adv., 2019, 9, 33678

Received 23rd July 2019

Accepted 2nd October 2019

DOI: 10.1039/c9ra05677g

rsc.li/rsc-advances

\section{Molecular imprinting on PtPd nanoflowers for selective recognition and determination of hydrogen peroxide and glucose $\dagger$}

\begin{abstract}
Caini Fan, ${ }^{a}$ Junjia Liu, ${ }^{b}$ Haiying Zhao, ${ }^{a}$ Ling Li, ${ }^{a}$ Min Liu, ${ }^{\star a}$ Jing Gao ${ }^{b}$ and Li Ma (DD *b
PtPd nanoflowers (PtPd NFs) exhibit intrinsic peroxidase-like activity as nanozymes, but the nanozymes lack substrate specificity and have low catalytic activity. Herein, a molecularly imprinted nanogel on PtPd NFs was prepared by using 3,3',5,5'-tetramethylbenzidine (TMB) as the template through the aqueous precipitation polymerization method. After the TMB was washed out, many substrate binding pockets were retained in the PtPd NFs. Scanning electron microscopy (SEM), transmission electron microscopy (TEM) and powder X-ray diffraction (XRD) were employed to characterize the molecularly imprinted polymer (MIP) PtPd nanoflowers (T-MIP-PtPd NFs). The obtained T-MIP-PtPd NFs exhibited enhanced catalytic activity and specific recognition for TMB. Compared with PtPd NFs, T-MIP-PtPd NFs showed a linear range from $0.01-5000 \mu \mathrm{M}$ and a detection limit of $0.005 \mu \mathrm{M}$ toward the detection of $\mathrm{H}_{2} \mathrm{O}_{2}$. Glucose can also be sensitively detected through cascade reaction by the T-MIP-PtPd NFs and glucose oxidase. Therefore, molecular imprinting on nanozymes technology shows promising application in biocatalysis and sensing fields.
\end{abstract}

\section{Introduction}

Nanozymes, a nanomaterial with enzyme-like activity, are attracting more and more attention thanks to their outstanding properties. ${ }^{1-3}$ To date, various nanozymes, including metalorganic frameworks (MOFs), ${ }^{4-7}$ metal nanoparticles, ${ }^{8}$ oxidized metal nanoparticles ${ }^{9,10}$ and some other nanomaterials, ${ }^{11}$ have been successfully prepared and used as enzyme mimics for catalytic applications. ${ }^{2}$ As one of the enzyme mimics, Pt nanoparticles have been demonstrated to exhibit versatile catalytic activity like peroxidase, catalase and superoxide dismutase., ${ }^{2,12}$ Recent studies reported that the Pt-based bimetallic nanoparticles (i.e. PtPd nanodendrites) can exhibit peroxidase-like activity. ${ }^{13,14}$ For this kind of nanozyme, the creation of mesoporous nanostructures is considered to be an effective method to improve the enzyme mimetic activity. ${ }^{14}$ With the presence of a mesoporous structure, specific surface area and accessible pores of the Pt-based nanozymes will be increased, and the activity will be improved. Recently, a surfactant-directing method for synthesizing multimetallic spheres with ultralarge mesoporous was reported ${ }^{15,16}$ and the obtained

${ }^{a}$ Department of Hypertension, Henan Provincial People's Hospital, Zheng Zhou, 450003, China.E-mail: liumin136@126.com

${ }^{b}$ School of Chemical Engineering and Technology, Hebei University of Technology, Tianjin 300130, China. E-mail: malio502@hebut.edu.cn

$\dagger$ Electronic supplementary information (ESI) available: Color evolution of TMB and ABTS in different reaction systems, optimization of experimental parameters, the selectivity of glucose detection. See DOI: $10.1039 / \mathrm{c} 9 \mathrm{ra} 05677 \mathrm{~g}$ mesoporous multimetallic spheres exhibited enhanced electrochemical activity thanks to the high surface area and mesoporous structure. Despite the progress made in the synthesis of multi-metal nanospheres, their enzyme mimetic properties are rarely reported and the mechanism remains elusive. In addition, there are still disadvantages for these nanozymes, such as complicated synthesis process, low specificity and low catalytic activity. ${ }^{1}$ Thus, the synthesis of the PtPd bimetallic nanomaterials with high peroxidase-mimic activity and substrate specificity are still challenges for further investigation.

Compared to natural enzymes, nanozymes are stable to denaturation, low in cost, highly resistant to high concentrations of substrates, flexible storage conditions and easy to manipulate catalytic activity. However, most nanozymes have no substrate specificity and can catalyze a lot of substrates leading to the lack of molecular recognition function. Molecularly imprinted polymers (MIPs) could create functional polymer materials with selective recognition properties. ${ }^{17}$ MIPs are usually prepared by bulk polymerization, photopolymerization, surface grafting polymerization and sol-gel mechanisms to generate imprinting specific cavities. ${ }^{18,19}$ Thus, combining MIPs with nanozymes provides a new class of nanozymes with improved selectivity and activity. ${ }^{20-22}$ For example, Zhang et al. ${ }^{23}$ imprinted substrate binding pockets gels on three different nanozymes and the experimental results showed that the molecular imprinting on nanozymes remarkably increased the selectivity and catalytic activity.

Thus, in this study, PtPd nanoflowers (PtPd NFs) were synthesized by a surfactant-directing method. Then, the MIP 
layer was created on the surface of PtPd NFs and the obtained nanocomposites were named as T-MIP-PtPd NFs. The structure and composition of PtPd and T-MIP-PtPd were analyzed by scanning electron microscopy (SEM), transmission electron microscope (TEM), energy dispersive X-ray spectroscopy (EDX) and powder X-ray diffraction (XRD). Their intrinsic peroxidaselike activities were investigated. The T-MIP-PtPd NFs was used to detect $\mathrm{H}_{2} \mathrm{O}_{2}$ and glucose through a sensitive colorimetric method. To the best of our knowledge, it is the first report regarding the preparation of TMB imprinted PtPd NFs and its application in detecting hydrogen peroxide and glucose.

\section{Materials and methods}

\section{Chemicals}

Chloroplatinic acid $\left(\mathrm{H}_{2} \mathrm{PtCl}_{6}\right)$ was purchased from Shanghai Macklin Biochemical Co., Ltd. (Shanghai, China). Potassium tetrachloroplatinate(II) $\left(\mathrm{K}_{2} \mathrm{PtCl}_{4}\right), \quad$ sodium tetrachloropalladate(II) $\left(\mathrm{Na}_{2} \mathrm{PdCl}_{4}\right)$, ascorbic acid, 3,3,5,5-tetramethylbenzidine (TMB), 2,2'-azinobis-(3-ethylbenzthiazoline-6sulphonate) (ABTS), F127 and glucose oxidase (GOD) were purchased from Shanghai Aladdin Bio-Chem Technology Co., Ltd. (Shanghai, China). Glacial acetic acid, anhydrous sodium acetate, and ethanol were purchased from Tianjin Fengchuan Chemical Reagent Technologies Co., Ltd.

\section{Preparation of PtPd NFs}

Typically, the solutions of $\mathrm{H}_{2} \mathrm{PtCl}_{6}(20 \mathrm{mM}, 1.2 \mathrm{~mL}), \mathrm{K}_{2} \mathrm{PtCl}_{4}$ $(20 \mathrm{mM}, 1.8 \mathrm{~mL})$ and $\mathrm{Na}_{2} \mathrm{PdCl}_{4}(20 \mathrm{mM}, 0.6 \mathrm{~mL})$ were mixed uniformity in a $10 \mathrm{~mL}$ centrifuge tube. Then F127 (60 mg) and HCl solution ( $6 \mathrm{M}, 60 \mu \mathrm{L}$ ) were added. After F127 was completely dissolved, ascorbic acid (0.1 M, $3 \mathrm{~mL})$ was added with ultrasonic at $40{ }^{\circ} \mathrm{C}$. After 4 hours, the obtained PtPd NFs were centrifuged and washed with Milli-Q water and ethanol, respectively.

\section{Molecular imprinting on PtPd NFs}

The aqueous precipitation polymerization method is used to prepare TMB imprinted PtPd NFs (T-MIP-PtPd NFs). Typically, PtPd NFs $\left(1 \mathrm{mg} \mathrm{mL}^{-1}, 0.5 \mathrm{~mL}\right)$ and TMB solution $(7.5 \mathrm{mM}, 20$ $\mu \mathrm{M})$ were mixed in HEPES buffer solution (20 mM, $\mathrm{pH} 7.6)$ that was purged with $\mathrm{N}_{2}$. Acrylamide $(2.9 \mathrm{mg}, 42 \mu \mathrm{M}), N$-isopropylacrylamide (NIPAAm) (4.6 mg, $42 \mu \mathrm{M}), N, N^{\prime}$-methylenebisacrylamide (MBAAm as crosslinker) $(2.4 \mathrm{mg}, 16 \mu \mathrm{M})$ and SDS ( $0.8 \mathrm{mg}$ ) were dissolved in $0.5 \mathrm{~mL}$ HEPES buffer solution to prepare a monomer solution. Polymerization of the monomer solutions was initiated by adding ammonium persulfate $(0.2$ $\mathrm{mg})$ and tetramethyl-ethylenediamine $(0.3 \mu \mathrm{L})$. The monomer and the PtPd NFs-containing solutions were mixed after $20 \mathrm{~min}$ of initiation, and then the mixture was continued to react for $1 \mathrm{~h}$ at room temperature. The resulting imprinted gels were collected by centrifugation at $5000 \mathrm{rpm}$ for $5 \mathrm{~min}$. Then $1 \mathrm{mM}$ $\mathrm{H}_{2} \mathrm{O}_{2}$ was added to react with the imprinted substrates so they can be easily removed by subsequent washing. As a control experiment, non-imprinted PtPd NFs nanozymes (NIP-PtPd NFs) were also prepared in the same way except that no substrate template was added.

\section{Determination of peroxidase-like activity of PtPd NFs, NIP-} PtPd NFs and T-MIP-PtPd NFs

Typically, $10 \mu \mathrm{L}\left(0.1 \mathrm{mg} \mathrm{mL} \mathrm{m}^{-1}\right)$ nanozymes (PtPd NFs, NIP-PtPd NFs and T-MIP-PtPd NFs) and $50 \mu \mathrm{L}$ of substrate $(0.375 \mathrm{mM}$, TMB or ABTS) were added to $0.93 \mathrm{~mL}$ HAc-NaAc buffer solution (0.05 M, pH 4.0) containing $10 \mu \mathrm{L} \mathrm{H}_{2} \mathrm{O}_{2}(0.2 \mathrm{M})$. The reaction mixture was incubated at $25{ }^{\circ} \mathrm{C}$ for $10 \mathrm{~min}$, and the peroxidaselike activity was investigated by detecting the absorbance (652 $\mathrm{nm}$ for TMB and $420 \mathrm{~nm}$ for ABTS). To measure the steadystate kinetics, various concentrations of substrates (TMB and ABTS) were used, respectively. For colorimetric detection, $0.1 \mathrm{mg} \mathrm{mL}^{-1}$ of nanozymes (PtPd NFs, NIP-PtPd NFs and T-MIPPtPd NFs) and $0.1 \mathrm{mM}$ of $\mathrm{H}_{2} \mathrm{O}_{2}$ were added to $9.3 \mathrm{~mL}$ acetate buffer solution (0.1 M, pH 4) containing substrates (TMB and ABTS) with various concentrations for $10 \mathrm{~min}$ at $25^{\circ} \mathrm{C}$. The kinetic parameters were calculated by the Michaelis-Menten equation (eqn (1)):

$$
\frac{1}{v}=\frac{K_{\mathrm{m}}}{V_{\max }}\left(\frac{1}{[\mathrm{~S}]}+\frac{1}{K_{\mathrm{m}}}\right)
$$

where $[\mathrm{S}]$ is the concentrations of TMB or ABTS.

\section{Detection of $\mathrm{H}_{2} \mathrm{O}_{2}$ and glucose}

The detection of $\mathrm{H}_{2} \mathrm{O}_{2}$ by a colorimetric assay was carried out as follows: $10 \mu \mathrm{L}$ of nanozyme $\left(0.1 \mathrm{mg} \mathrm{mL}^{-1}\right)$ (PtPd NFs, NIP-PtPd NFs and T-MIP-PtPd NFs), $50 \mu \mathrm{L}$ of TMB (7.5 mM), and $10 \mu \mathrm{L}$ of $\mathrm{H}_{2} \mathrm{O}_{2}(0.2 \mathrm{M})$ were added into $930 \mu \mathrm{L}$ of HAc-NaAc buffer solution (0.05 M, pH 4.0). Afterward, the absorbance was measured at $652 \mathrm{~nm}$ after $15 \mathrm{~min}$ of incubation at room temperature. For comparison, ABTS $(50 \mu \mathrm{L} 7.5 \mathrm{mM})$ was also used as colorimetric substrate to detect $\mathrm{H}_{2} \mathrm{O}_{2}$, and the absorbance was measured at $420 \mathrm{~nm}$ after $15 \mathrm{~min}$ of incubation at room temperature.

PtPd NFs, NIP-PtPd NFs and T-MIP-PtPd NFs were also used to detect glucose through catalyzing the production of $\mathrm{H}_{2} \mathrm{O}_{2}$ from the oxidation of glucose by glucose oxidase. In a typical process, $300 \mu \mathrm{L}$ of PBS (phosphate buffer solution, $\mathrm{pH} 7.0$ ) containing a serials concentration of glucose and $100 \mu \mathrm{L}$ of PBS

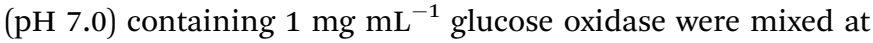
$37{ }^{\circ} \mathrm{C}$ for $30 \mathrm{~min}$. Then $540 \mu \mathrm{L}$ of HAc-NaAc buffer solution, 50 $\mu \mathrm{L}$ of TMB $(0.35 \mathrm{mM})$ and $10 \mu \mathrm{L}$ of $\left(0.1 \mathrm{mg} \mathrm{mL}^{-1}\right)$ nanozymes (PtPd NFs, NIP-PtPd NFs and T-MIP-PtPd NFs) were added to the above mixture, and incubated at $25{ }^{\circ} \mathrm{C}$ for $10 \mathrm{~min}$ to oxidize the reaction solution from colorless to blue.

\section{Results and discussion}

\section{Characterization of T-MIP-PtPd NFs}

As shown in Fig. 1, PtPd NFs were synthesized firstly and then the monomers including acrylamide, NIPAAm and MBAAm were mixed with PtPd NFs to perform precipitation polymerization. TMB was used as a template for imprinting. After the TMB imprinted polymer layer was formed on the PtPd NFs surface, the TMB molecules were washed away, leaving behind the binding pockets that can selectively rebinding TMB. As shown in Fig. 2A and C, SEM and TEM images of PtPd NFs 


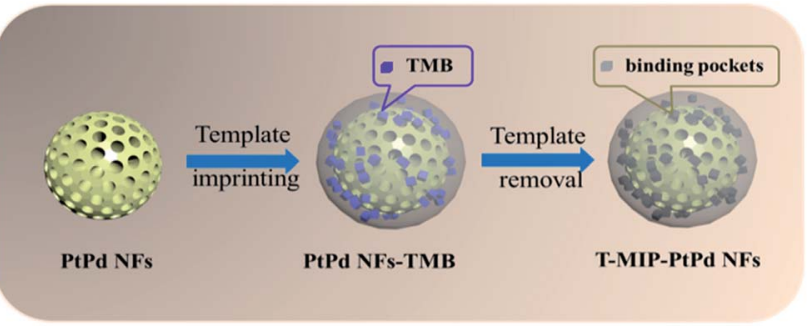

Fig. 1 The schematic diagram of preparing T-MIP-PtPd NFs.

indicated a spherical nanostructure with flower-like morphology and mesoporous on the surface. The particle size of PtPd NFs was approximately $120 \mathrm{~nm}$ (Fig. 2A and C). Compared to PtPd NFs, T-MIP-PtPd NFs had a bigger size due to the polymer coating on the surface of PtPd NFs (Fig. 2B and D). XRD pattern of T-MIP-PtPd NFs showed four strong diffraction peaks at $39.7^{\circ}, 46.4^{\circ}, 67.5^{\circ}$ and $81.2^{\circ}$, which can be assigned to the (111), (200), (220) and (311) crystalline planes of the standard pattern of Pt and Pd, respectively ${ }^{16,24}$ (Fig. 2E). EDX analysis of the T-MIP-PtPd NFs showed obvious Pt, Pd, C, N and O peaks
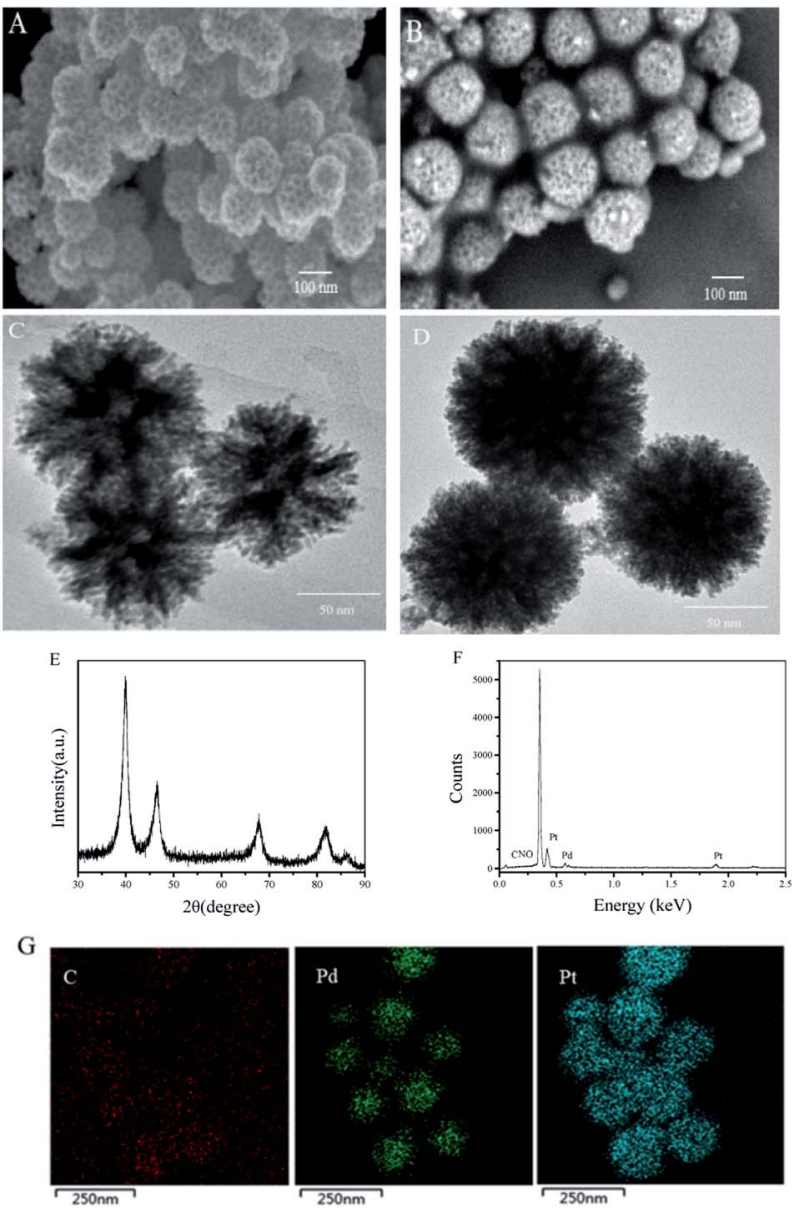

Fig. 2 SEM images of (A) PtPd NFs, (B) T-MIP-PtPd NFs, TEM images of (C) PtPd NFs, (D) T-MIP-PtPd NFs, (E) XRD pattern of T-MIP-PtPd NFs, (F) EDX spectrum of T-MIP-PtPd NFs and (G) elemental mapping images of T-MIP-PtPd NFs.
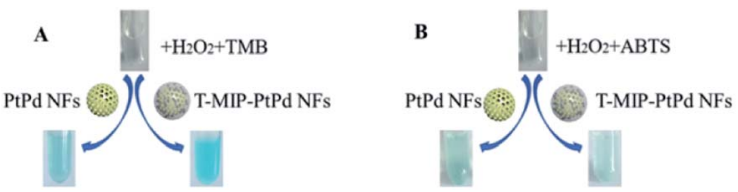

Fig. 3 Photographs with color change showing the activity and specificity of T-MIP-PtPd NFs for oxidation of (A) TMB and (B) ABTS with $\mathrm{H}_{2} \mathrm{O}_{2}$.

(Fig. 2F). Moreover, elemental mapping images of T-MIP-PtPd NFs (Fig. 2G) showed that C, Pt and Pd element were homogeneously distributed through the T-MIP-PtPd NFs structure. The content of $\mathrm{C}$ element is much lower than $\mathrm{Pt}$ and $\mathrm{Pd}$, and the boundary between $\mathrm{C}$ and $\mathrm{Pt}$ (and Pd) is unclear, indicating the polymer layer coating on the PtPd NFs is thin.

\section{Peroxidase-like activity of T-MIP-PtPd NFs}

The peroxidase activity of PtPd NFs, NIP-PtPd NFs and T-MIPPtPd NFs was investigated at $25{ }^{\circ} \mathrm{C}$ using TMB as the substrate (Fig. S1†). Besides, similar reaction was also carried out using ABTS as reductant (Fig. S2 $\dagger$ ). These results confirmed that PtPd NFs, NIP-PtPd NFs and T-MIP-PtPd NFs were peroxidase mimicking nanozyme (Fig. 3A and B). The mechanism for the oxidation of TMB may be as follows: firstly, the d-band of platinum will become nearly bare when palladium atoms combine with platinum atoms. Then, the lone electron pairs of $\mathrm{H}_{2} \mathrm{O}_{2}$ will fill the d-band. After that, $\mathrm{H}_{2} \mathrm{O}_{2}$ cleave into -OH, and $-\mathrm{OH}$ can oxidize TMB to TMB $+{ }^{25}$ Although both TMB and ABTS can be oxidized by T-MIP-PtPd NFs, it showed poor catalytic activity for ABTS.

\section{Imprinting enhanced the activity of nanozyme}

To compare the catalytic activity of PtPd NFs, NIP-PtPd NFs and T-MIP-PtPd NFs, the value changes in absorption spectra during the catalytic process was measured using UV-vis
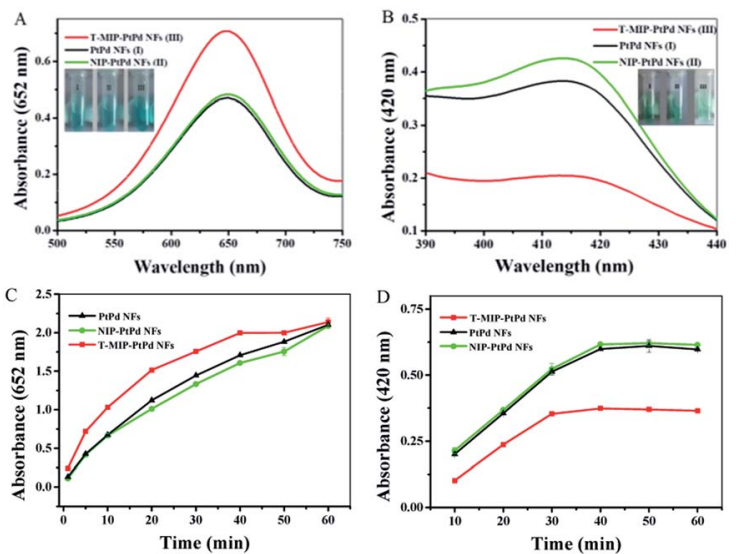

Fig. 4 UV-vis spectra after 30 min of reaction for (A) TMB and (B) ABTS oxidation, inset: photographs with color change showing peroxidaselike enzyme activity, the kinetics of (C) TMB oxidation monitored at $652 \mathrm{~nm}$ and (D) ABTS oxidation monitored at $420 \mathrm{~nm}$ by PtPd NFs, NIP-PtPd NFs and T-MIP-PtPd NFs. 

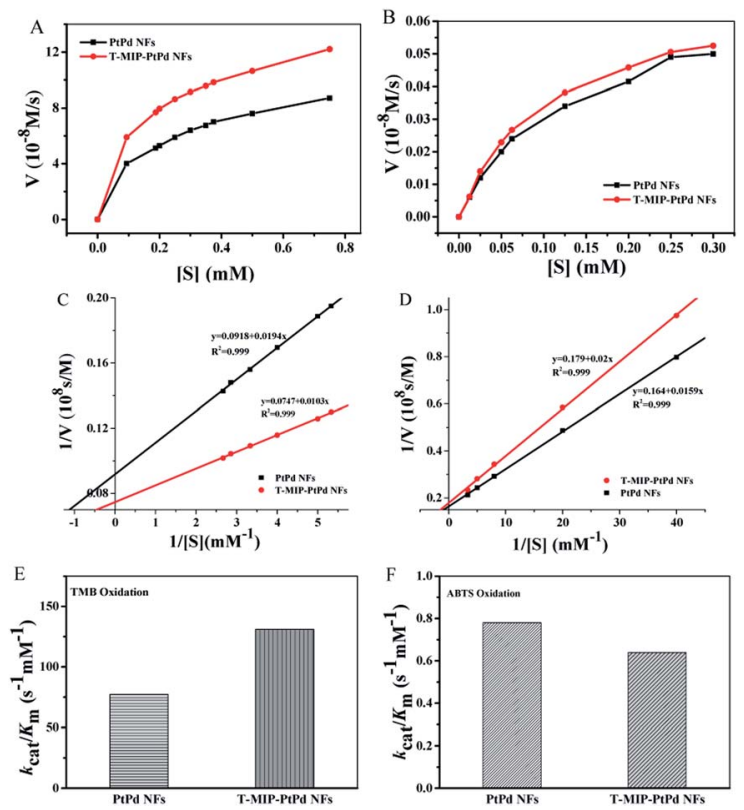

Fig. 5 Reaction rates with various concentrations of (A) TMB and (B) ABTS, kinetic parameters for oxidizing (C) TMB and (D) ABTS, and the catalytic efficiency $\left(k_{\text {cat }} / K_{m}\right)$ of PtPd NFs and T-MIP-PtPd NFs for (E) TMB oxidation and (F) ABTS oxidation.

spectrophotometer. When PtPd NFs was used as catalyst, the absorbance at $652 \mathrm{~nm}$ reached 0.47 after reaction for $10 \mathrm{~min}$ (Fig. 4A, black trace). In contrast, when T-MIP-PtPd NFs were used as catalyst, the absorbance reached 0.71 (Fig. $4 \mathrm{~A}$, red trace), indicating the enhanced activity. According to these results, T-MIP-PtPd NFs could enhance the catalytic activity for TMB oxidation. The improved catalytic activity can be attributed to the TMB pockets, which imprinted in the PtPd NFs and had selective recognition for TMB molecules (Fig. 4C). Moreover, the increased local substrate concentration after MIP may also enhance the catalytic rate according to the mass action law. ${ }^{26}$ The system of NIP-PtPd NFs with TMB and $\mathrm{H}_{2} \mathrm{O}_{2}$ exhibited similar absorption value to PtPd NFs system (Fig. 4A, green trace), indicating similar catalytic activity of NIP-PtPd NFs and PtPd NFs. Because the polymer layer around the PtPd NFs was thin, the polymer layer might have no obvious influence on the activity. In contrast, when ABTS was used as another substrate to investigate the selectivity of T-MIP-PtPd NFs, the catalytic activity of T-MIP-PtPd NFs for ABTS was lower than that of PtPd NFs and NIP-PtPd NFs (Fig. 4B and D). Therefore, after TMB imprinting, the selectivity of T-MIP-PtPd NFs was improved for TMB oxidation and inhibited for ABTS oxidation. In order to achieve an optimal colorimetric effect, the effect of $\mathrm{pH}$, temperature, reaction time, $\mathrm{H}_{2} \mathrm{O}_{2}$ concentration, and quantity of T-MIP-PtPd NFs on relative activity were investigated (Fig. S4 $\dagger$ ). The highest absorbance was reached when $0.1 \mathrm{mg}$ $\mathrm{mL}^{-1}$ of T-MIP-PtPd NFs, $0.35 \mathrm{mM} \mathrm{TMB}$, and $2.0 \mathrm{M} \mathrm{H}_{2} \mathrm{O}_{2}$ were used. Therefore, the above condition was selected in the following experiments.

\section{Kinetic analysis}

In order to investigate the mechanism of peroxidase-mimetic activity, the reaction kinetics of PtPd NFs and T-MIP-PtPd NFs were measured (Fig. 5A and B). Reaction rates with PtPd NFs and T-MIP-PtPd NFs as catalysts were tested by varying the concentration of TMB while fixing $\mathrm{H}_{2} \mathrm{O}_{2}$ concentration. For comparison, $K_{\mathrm{m}}$ of free HRP was also determined. The $K_{\mathrm{m}}$ value for PtPd NFs and T-MIP-PtPd NFs with TMB as substrate was $0.211 \mathrm{mM}$ and $0.137 \mathrm{mM}$, respectively (Fig. 5C and D). The $K_{\mathrm{m}}$ value for T-MIPPtPd NFs is much lower than those of PtPd NFs and free HRP (Table 1), indicating that T-MIP-PtPd NFs had stronger affinity to TMB than PtPd NFs and free HRP. However, the $K_{\mathrm{m}}$ for T-MIPPtPd NFs with ABTS as substrate was higher than that of PtPd NFs, implying that the imprinting process only offer accessible site for TMB and then enhance substrate specificity.

Using TMB as substrate, the catalytic efficiency $\left(k_{\text {cat }} / K_{\mathrm{m}}\right)$ of PtPd NFs and T-MIP nanozymes were also investigated. Compared to PtPd NFs, the $k_{\text {cat }}$ value of the T-MIP-PtPd NFs increased by 0.2 -fold, while the $K_{\mathrm{m}}$ value dropped by $10 \%$ for
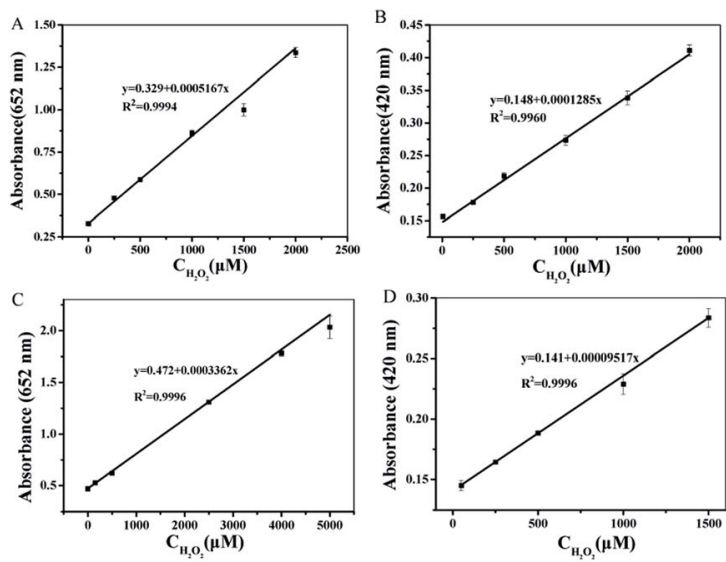

Fig. 6 Linear calibration plot for $\mathrm{H}_{2} \mathrm{O}_{2}$ with different concentration in absorbance of $652 \mathrm{~nm}$ reacted with (A) PtPd NFs and TMB, (B) PtPd NFs and ABTS, (C) T-MIP-PtPd NFs and TMB, (D) T-MIP-PtPd NFs and ABTS

Table 1 Comparison of catalytic parameters of the PtPd NFs, T-MIP-PtPd NFs and HRP with TMB and ABTS as substrate

\begin{tabular}{llllll}
\hline Nanozyme & Substrate & $K_{\mathrm{m}}(\mathrm{mM})$ & $V_{\max }\left(10^{-8} \mathrm{M} \mathrm{s}^{-1}\right)$ & $k_{\text {cat }}\left(\mathrm{s}^{-1}\right)$ & 16.28 \\
\hline PtPd NFs & TMB & 0.211 & 10.87 & 17.94 & 77.2 \\
T-MIP-PtPd NFs & TMB & 0.137 & 13.30 & - & 131 \\
HRP & TMB & 0.434 (ref. 27) & 10.00 (ref. 27) & - \\
PtPd NFs & ABTS & 0.097 & 6.180 & 0.0756 \\
T-MIP-PtPd NFs & ABTS & 0.112 & 5.603 & 0.0715
\end{tabular}


Table 2 Comparison of different $\mathrm{H}_{2} \mathrm{O}_{2}$ sensors in terms of linear range and LOD

\begin{tabular}{lllll}
\hline Materials & Substrate & Linear range $(\mu \mathrm{M})$ & LOD $(\mu \mathrm{M})$ \\
\hline $\mathrm{Fe}_{3} \mathrm{O}_{4} @ \mathrm{C}$ YSNs & TMB & $1-20$ & 0.39 & Reference \\
PtPd NDs/GNs & TMB & $0.5-150$ & 0.1 \\
PtPd NRs & TMB & $0.02-50000$ & 0.0086 \\
HRP & TMB & 0.43 & 3.70 & 13 \\
PtPd NFs & TMB & $0.05-2000$ & 0.02 & 29 \\
T-MIP-PtPd NFs & TMB & $0.01-5000$ & 0.005
\end{tabular}

ABTS oxidation. We plotted $k_{\text {cat }} / K_{\mathrm{m}}$ of T-MIP-PtPd NFs for TMB and ABTS oxidation to compare PtPd NFs (Fig. 5E and F). The catalytic efficiency of T-MIP-PtPd NFs showed 0.7-fold higher than that of PtPd NFs for oxidizing TMB, but 0.15-fold lower for oxidizing ABTS. The imprinting in PtPd NFs can selectively enrich TMB substrate near the T-MIP-PtPd NFs and then result in enhanced catalytic activity.

\section{Detection of $\mathrm{H}_{2} \mathrm{O}_{2}$}

Under the optimized experimental conditions (see ESI $\dagger$ ), a series concentration of $\mathrm{H}_{2} \mathrm{O}_{2}$ were detected based on the peroxidase-like activity of T-MIP-PtPd NFs through colorimetric method. When TMB was used as substrate, the absorbance of oxidized TMB is linearly correlated to concentration of $\mathrm{H}_{2} \mathrm{O}_{2}$ from $0.05-2000 \mu \mathrm{M}$ (limit of detection (LOD) was $0.02 \mu \mathrm{M}$ ) catalyzed by PtPd NFs (Fig. 6A). The absorbance ranged from 0.01-5000 $\mu \mathrm{M}$ (LOD was $0.005 \mu \mathrm{M}$ ) catalyzed by T-MIP-PtPd NFs. This result proved that molecular imprinted nanozyme enhances its activity thanks to the pockets of TMB molecule (Fig. 6C). When ABTS was used as the substrate, the linear range between the absorbance and concentration of $\mathrm{H}_{2} \mathrm{O}_{2}$ was ranged from 0.05-2000 $\mu \mathrm{M}$ by PtPd NFs (Fig. 6B). For T-MIP-PtPd NFs, the linear range for $\mathrm{H}_{2} \mathrm{O}_{2}$ sensing was ranged from $0.01-1500$
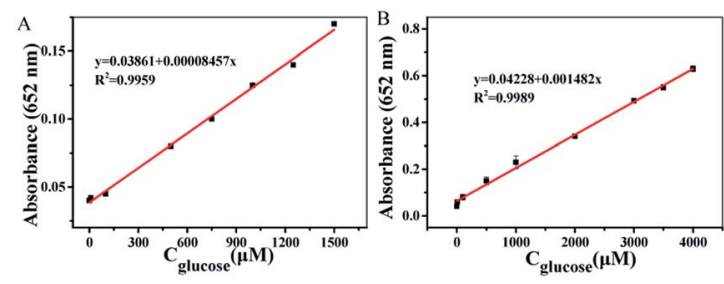

Fig. 7 Linear calibration plot for glucose with different concentration of glucose in absorbance of $652 \mathrm{~nm}$ with (A) PtPd NFs and (B) T-MIPPtPd NFs. $\mu \mathrm{M}$ (Fig. 6D), indicating that the special pockets of TMB will hinder the approach of ABTS molecule. It can be seen from Table 2 that detection of $\mathrm{H}_{2} \mathrm{O}_{2}$ based on the T-MIP-PtPd NFs exhibited good performance, which further confirmed that the molecular imprinted method can enhance the catalytic activity.

\section{Detection of glucose}

Through combining T-MIP-PtPd NFs and GOx, we further developed a colorimetric method for the determination of glucose. Glucose can be catalyzed by GOx to generate gluconic acid and $\mathrm{H}_{2} \mathrm{O}_{2}$. In the presence of $\mathrm{H}_{2} \mathrm{O}_{2}$, TMB can be oxidized through the catalytic action of peroxidase-like nanozymes. The combination of two catalytic reactions was used to detect glucose through colorimetric method. With the increasing concentrations of glucose, the absorbance will increase from 0.05 to $1500 \mu \mathrm{M}$ and a LOD of $0.023 \mu \mathrm{M}$ for PtPd NFs was obtained (Fig. 7A). The T-MIP-PtPd NFs exhibits a linear detection range from 0.02 to $4000 \mu \mathrm{M}$ and a LOD of $0.017 \mu \mathrm{M}$ (Fig. 7B). Compared with the reported glucose sensors, T-MIP-PtPd NFs showed better catalytic performance for detecting glucose owing to the molecularly imprinted process (Table 3). This efficient detection performance was mainly ascribed to: (i) the T-MIP-PtPd NFs had excellent selectivity to TMB; (ii) the T-MIPPtPd NFs provided an optimal microenvironment to stabilize GOx.

To evaluate the selectivity of the established method for glucose detection, the influence of lactose, galactose, fructose, and arabinose were investigated (Fig. S5 $\dagger$ ). All above interferences showed negligible effect on glucose detection. This phenomenon demonstrates that the reaction system is highly selective for glucose. The reproducibility of the T-MIP-PtPd NFs was investigated by analyzing $0.6 \mathrm{mM}$ glucose for six times in parallel. The relative standard deviation (RSD) was $4.8 \%$, which indicates that the testing results had acceptable reproducibility. To verify the feasibility of the as-prepared colorimetric method,

Table 3 Comparison of colorimetric detection of glucose with different nanomaterials

\begin{tabular}{|c|c|c|c|c|}
\hline Materials & Substrate & Linear range $(\mu \mathrm{M})$ & LOD $(\mu \mathrm{M})$ & Reference \\
\hline PB NPs & ABTS & $0.1-50$ & 0.03 & 30 \\
\hline PB/MIL-101(Fe) & TMB & $0.1-1000$ & 0.04 & 4 \\
\hline Au NPs & TMB & $18-1100$ & 4 & 31 \\
\hline PtPd NFs & TMB & $0.05-1500$ & 0.023 & This article \\
\hline T-MIP-PtPd NFs & TMB & $0.02-4000$ & 0.017 & This article \\
\hline
\end{tabular}


glucose concentration in human serum samples were measured by the standard addition method (ESI $\dagger$ ). All the obtained results were listed in Table S1.† It was found that the colorimetric method has a favorable recovery of $92.3-98.7 \%$ to detect glucose, which indicates that T-MIP-PtPd NFs, TMB and $\mathrm{H}_{2} \mathrm{O}_{2}$ system could be reliable for glucose detection in the biological environment.

\section{Conclusions}

In summary, molecular imprinting PtPd nanoflowers were successfully fabricated. The integration of PtPd nanozyme and molecular imprinting can enhance the peroxidase like activity and specificity of T-MIP-PtPd NFs. The obtained T-MIP-PtPd NFs exhibit better catalytic efficiency than that of PtPd NFs and HRP. Based on T-MIP-PtPd NFs, colorimetric sensor was developed for detecting hydrogen peroxidase and glucose. The T-MIP-PtPd showed better catalytic performance as peroxidase mimetic in the optical detection of hydrogen peroxidase and glucose. Thanks to these benefits, T-MIP-PtPd NFs provides a facile, low-cost, sensitive and selective method for colorimetric detection of $\mathrm{H}_{2} \mathrm{O}_{2}$ and glucose molecules. With good enzyme-like activity and specificity, the method of combining molecular imprinting with nanozyme can be extended to fabricate other kinds of nanozymes.

\section{Conflicts of interest}

There are no conflicts to declare.

\section{Acknowledgements}

This work was supported by Science and technology projects in Henan province (182102311235), the National Natural Science Foundation of China (No. 21576068, 21276060 and 21878068), the Natural Science Foundation of Hebei Province, China (B2017202056), the Program for Top 100 Innovative Talents in Colleges and Universities of Hebei Province, China (SLRC2017029) and Hebei High level personnel of support program, China (A2016002027).

\section{Notes and references}

1 D. Jiang, D. Ni, Z. T. Rosenkrans, et al., Chem. Soc. Rev., 2019, 48, 3683-3704.

2 H. Wei and E. Wang, Chem. Soc. Rev., 2013, 42, 6060-6093. 3 S. Jin, C. Wu, Z. Ye, et al., Sens. Actuators, B, 2019, 283, 18-34. 4 F. Cui, Q. Deng and L. Sun, RSC Adv., 2015, 5, 98215-98221.
5 Y. Zhang, C. Dai, W. Liu, et al., Microchim. Acta, 2019, 186, 340-349.

6 W. Xu, L. Jiao, H. Yan, et al., ACS Appl. Mater. Interfaces, 2019, 25, 22096-22101.

7 Z. Zhao, T. Lin, W. Liu, et al., Spectrochim. Acta, Part B, 2019, 219, 240-247.

8 X. Liu, X. Wang, C. Qi, et al., Appl. Surf. Sci., 2019, 479, 532539.

9 X. Cheng, L. Huang, X. Yang, et al., J. Colloid Interface Sci., 2019, 535, 425-435.

10 X. Cao and N. Wang, Analyst, 2011, 136, 4241-4246.

11 X. Wang, Y. Hu and H. Wei, Inorg. Chem. Front., 2016, 3, 4160.

12 T. Yu, W. Wang, J. Chen, et al., J. Phys. Chem. C, 2012, 116, 10516-10521.

13 X. Chen, B. Su, Z. Cai, et al., Sens. Actuators, B, 2014, 201, 286-292.

14 S. Ge, W. Liu, H. Liu, et al., Biosens. Bioelectron., 2015, 71, 456-462.

15 B. Jiang, C. Li, M. Imura, et al., Adv. Sci., 2015, 2, 1500112.

16 L. Ma, L. Zhou, Y. He, et al., Electroanalysis, 2018, 30, 18011810.

17 M. Dinc, C. Esen and B. Mizaikoff, TrAC, Trends Anal. Chem., 2019, 114, 202-217.

18 H. Zhang, Adv. Mater., 2019, e1806328.

19 M. Zarejousheghani, W. Lorenz, P. Vanninen, et al., Polymers, 2019, 11, 888-905.

20 W. Zhao, H. Yang, S. Xu, et al., Colloids Surf., A, 2018, 555, 95-102.

21 T. Kajisa and T. Sakata, ACS Appl. Mater. Interfaces, 2018, 10, 34983-34990.

22 D. Duan, H. Yang, Y. Ding, et al., Electrochim. Acta, 2018, 261, 160-166.

23 Z. Zhang, X. Zhang, B. Liu, et al., J. Am. Chem. Soc., 2017, 139, 5412-5419.

24 L. Wang and Y. Yamauchi, J. Am. Chem. Soc., 2013, 135, 16762-16765.

25 R. Mandal, A. Baranwal, A. Srivastava, et al., Biosens. Bioelectron., 2018, 117, 546-561.

26 Z. Zhang, Y. Li, X. Zhang, et al., Nanoscale, 2019, 11, 48544863.

27 V. K. Singh, P. K. Yadav, S. Chandra, et al., J. Mater. Chem. B, 2018, 6, 5256-5268.

28 N. Lu, M. Zhang, L. Ding, et al., Nanoscale, 2017, 9, 45084515.

29 J. Liu and Y. Lu, Angew. Chem., Int. Ed., 2005, 45, 90-94.

30 W. Zhang, D. Ma and J. Du, Talanta, 2014, 120, 362-367.

31 Y. Jv, B. Li and R. Cao, Chem. Commun., 2010, 46, 8017-8019. 